\title{
A Gallai-Type Transversal Problem in the Plane
}

\author{
Jürgen Eckhoff \\ Fachbereich Mathematik, Universität Dortmund, \\ W-4600 Dortmund 50, Federal Republic of Germany
}

\begin{abstract}
Let $\mathscr{K}$ be a family of compact convex sets in the plane. We show that if every three members of $\mathscr{K}$ admit a common line transversal, then there exist four lines which together meet all the members of $\mathscr{K}$.
\end{abstract}

\section{Introduction}

Let $\mathscr{K}$ be a family of compact convex sets in the plane. We say that $\mathscr{K}$ has property $T(r)$ if every $r$ or fewer members of $\mathscr{K}$ admit a line transversal, that is, a straight line which intersects these members (and possibly other members of $\mathscr{K}$ as well). We say that $\mathscr{K}$ has property $T$ if there exists a line transversal for all the members of $\mathscr{K}$. Such a line is also called a common 1-transversal, or a stabbing line for $\mathscr{K}$.

Over the years, considerable effort has been devoted to finding general conditions on the family $\mathscr{K}$ which assure that if $\mathscr{K}$ has property $T(r)$, for some fixed integer $r$, then $\mathscr{K}$ has property $T$. Such a result, if true, is said to be of Helly-type. We mention two prominent examples below, one going back to 1940, the other one dating from 1989. (For historical comments and a survey of the literature on transversals up to 1963, the reader is referred to [2]. Transversal problems are by no means restricted to the plane. A wealth of more recent results is documented in a new report by Goodman et al. [5].)

Our first example is a special case of a theorem of Santaló [13]. It asserts that, for any family of rectangles in the plane with sides parallel to the coordinate axes, $T(6)$ implies $T$. This is best possible in the sense that $T(6)$ cannot be replaced by $T(5)$ and that the conclusion fails for more general families of convex sets. The higher-dimensional version of Santalo's result deals with line (resp. hyperplane) transversals for families of parallelotopes in $R^{d}$. (For an extension in the latter case, see [8].) 
Our second example is supplied by the recent proof - due to Tverberg [14]- of a long-standing conjecture of Grünbaum [7]. This conjecture (now a theorem) claimed that, for any family of pairwise disjoint translates of a given compact convex set in the plane, $T(5)$ implies $T$. Again, simple examples show that the weaker assumption $T(4)$ would not suffice for the conclusion and that neither the disjointness hypothesis nor the requirement that the members be translates of one another can be dropped. We point out that Grünbaum's conjecture is far more difficult to prove than Santalo's result and, for that matter, most results in this area. (Compare the discussion in Section 4 of [5].) The conjecture had been verified earlier for disks (hence ellipses) by Danzer [1] and for squares (hence parallelograms) by Grünbaum [7]. A weaker form of the conjecture which assumed $T(128)$ instead of $T(5)$ had been established by Katchalski [11].

The above examples only serve to confirm the general rule that rather stringent conditions on the shape and the mutual position of the sets in $\mathscr{K}$ must be imposed in order to obtain a Helly-type transversal theorem. In this paper, therefore, we are concerned with transversal problems of a different nature (to be explained in a moment). We thereby continue our research on line transversals in the plane begun in [3] and [4].

As before, let $\mathscr{K}$ be a family of compact convex sets in the plane, and let $n$ be a positive integer. We say that $\mathscr{K}$ has property $T^{n}$ if $\mathscr{K}$ can be partitioned into $n$ or fewer subfamilies, each of which have a line transversal. In other words, $\mathscr{K}$ has property $T^{n}$ if $n$ lines in the plane whose union meets every member of $\mathscr{K}$ can be found. Occasionally, we say that $\mathscr{K}$ can be "stabbed" by those lines. Notice that $T^{1}$ is the same as $T$. The idea here is to make the "stabbing number" $n$ as small as possible while allowing the members of $\mathscr{K}$ to be quite general. This leads to a class of transversal problems called Gallai-type problems (see [2]-[5] and [12]). A variant has been considered by Wegner [15].

For each $r \geq 3$, the problem is to determine the smallest possible integer $n$ such that, for any family of compact convex sets in the plane, $T(r)$ implies $T^{n}$. It will be seen shortly that such an integer always exists but that its value is only interesting for $r=3$ and $r=4$. The problem was first studied by Hadwiger and Debrunner (see Proposition 26 of [10]) who dealt with the case $r=4$. To be precise, these authors restricted their attention to families of positive homothets (resp. translates) of a given compact convex set $K$. They proved that $T(4)$ implies $T^{4}$ for families of homothets of $K$, whereas it implies $T^{3}$ for families of translates of $K$. Later it turned out that even $T^{2}$ can be achieved and, moreover, the special assumptions on the shape of the sets can be dropped altogether. This shows

$$
T(4) \Rightarrow T^{2}
$$

Assertion (1.1) was proved by the author in 1969 (see [3] and [4]). As there are no Helly-type transversal theorems for general convex sets (i.e., $T(r) \nRightarrow T$ for $r \geq 3$ ), this settles the problem except for the case $r=3$ which to this day is still open. A family of 14 convex sets having property $T(3)$, but not $T^{2}$, is exhibited in [4]. It simplifies an earlier example of an infinite family with the same properties 
described in [3]. Hence we have

$$
T(3) \nRightarrow T^{2} .
$$

For some time, even the existence of a finite stabbing number for $T(3)$ families was in doubt but in $1975 \mathrm{Kramer}$ [12] was able to show that, in fact, T(3) implies $T^{5}$. This result appeared in Kramer's doctoral dissertation (see also p. 257 of [6]) and has not been published elsewhere. Our goal in this paper is to improve on Kramer's upper bound (while employing his main ideas) by showing that

$$
T(3) \Rightarrow T^{4} \text {. }
$$

In other words, we establish the following:

Theorem. If a family of compact convex sets in the plane is such that every three of its members admit a common line transversal, then the family can be stabbed by four lines.

We do not know whether the stabbing number "four" in this statement may be replaced by "three." 'For additional remarks, see the final section of this paper.

\section{Preliminaries}

Before proceeding to the proof of our theorem, we need to establish some geometric terminology and describe certain reductions of the problem which will facilitate the ensuing discussion.

In what follows, $\mathscr{K}$ is always a family of compact convex sets in the plane, and $n$ is a positive integer. The first reduction uses a compactness argument to show that it is sufficient to deal with finite families $\mathscr{K}$.

$$
\text { If every finite subfamily of } \mathscr{K} \text { has property } T^{n} \text {, then so has } \mathscr{K} \text {. }
$$

This was proved in a more general context in [3]. The proof is quite standard to anyone familiar with geometric transversals and is not given here.

The second reduction allows us to work with convex sets which have a nonempty interior. Strictly speaking, this reduction is not essential for the proof but it saves us some extra considerations. For $K$ a set in the plane and $\varepsilon$ a positive real number, let $K(\varepsilon)$ denote the Minkowski sum $K+B_{\varepsilon}$, where $B_{\varepsilon}$ is the closed disk of radius $\varepsilon$ centered at the origin. It is well known that if $K$ is compact and convex, then $K(\varepsilon)$ is compact, convex, and smooth; in particular, $K(\varepsilon)$ has interior points. Set $\mathscr{K}(\varepsilon):=\{K(\varepsilon) \mid K \in \mathscr{K}\}$. Then we have

$$
\text { If } \mathscr{K}(\varepsilon) \text { has property } T^{n} \text { for every } \varepsilon>0 \text {, then so has } \mathscr{K} \text {. }
$$

\footnotetext{
${ }^{1}$ In the Proceedings of the Second Colloquium on Discrete Geometry, Salzburg (1980), pp. 45-49, we claimed to have proved that $T(3) \Rightarrow T^{3}$. Unfortunately, our proof was in error.
} 


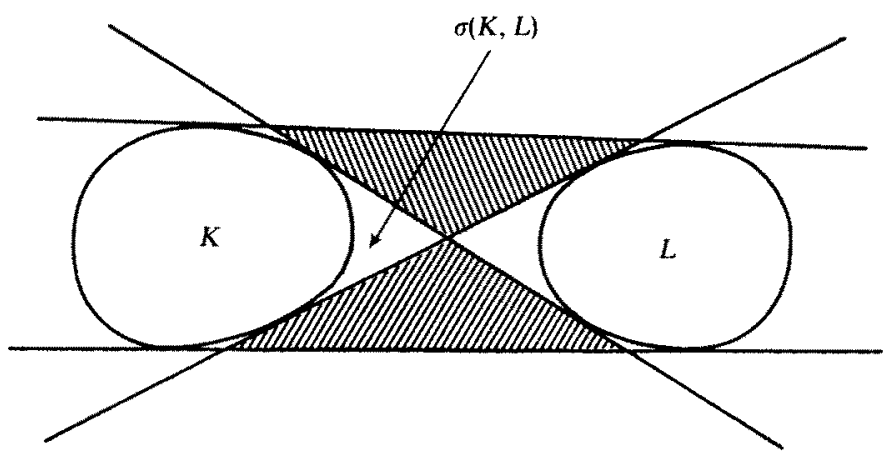

Fig. 1. The inner and outer common tangents of $K$ and $L$.

This is an easy consequence of assertion (2.1). Again, the proof is omitted.

Our last reduction is almost trivial. It states that in proving the main theorem we may as well assume that $\mathscr{K}$ contains pairs of disjoint sets. For, otherwise, $\mathscr{K}$ would have property $T$. To see this, project the members of $\mathscr{K}$ orthogonally onto some fixed line. If every two members intersect, then the same is true of the resulting segments. Thus, by Helly's theorem on the line, there is a point common to all these segments and so the projecting line corresponding to this point is a stabbing line for $\mathscr{K}$.

The following special terminology is used in this paper. Suppose $K$ and $L$ are two disjoint compact convex sets, both with interior points. There are exactly four lines in the plane which simultaneously support $K$ and $L$ (see Fig. 1). These lines are called the inner or outer common tangents of $K$ and $L$, depending on whether they separate the sets or not. ${ }^{2}$ The angle between the inner common tangents is denoted by $\sigma(K, L)$. To be precise, this is the interior angle of the closed double cone which contains $K$ and $L$ and is bounded by the inner tangents. This double cone is said to be associated with the pair $\{K, L\}$. The outer common tangents of $K$ and $L$ form two triangles (shaded in Fig. 1) with the inner common tangents. If these triangles are added to the double cone just mentioned, the resulting (closed) region is clearly the set of all points in the plane through which there passes a common line transversal of $K$ and $L$.

This observation leads at once to the following useful criterion. Suppose $K, L$, and $M$ are three pairwise disjoint compact convex sets (not necessarily with interior points). If there exist three lines $a, b$, and $c$ in the plane such that each of the sets is separated from the other two by one of these lines, while none of the lines intersects all three sets, then there is no line transversal for $K, L$, and $M$. (Incidentally, the converse is also true.) We refer to this condition by saying that $K, L$, and $M$ are separated by $a, b$, and $c$. The lines must then be distinct but they are allowed to meet in a point.

\footnotetext{
${ }^{2}$ In this paper, separation by lines is always understood to be weak separation.
} 


\section{Proof of the Theorem}

We are now ready to prove our main result.

Let $\mathscr{K}$ be a family of compact convex sets in the plane, and suppose $\mathscr{K}$ has property $T(3)$. We must show that $\mathscr{K}$ has property $T^{4}$. In view of the reductions described in the preceding section, we may assume that $\mathscr{K}$ is finite and that every member of $\mathscr{K}$ has a nonempty interior. Furthermore, we may assume that $\mathscr{K}$ contains at least one pair of disjoint sets. Among all such pairs choose one, say $\left\{K_{0}, L_{0}\right\}$, for which the interior angle $\sigma\left(K_{0}, L_{0}\right)$ defined in Section 2 is minimal. These assumptions imply that $\sigma\left(K_{0}, L_{0}\right)>0$ and that any member of $\mathscr{K}$ which lies in the double cone associated with $\left\{K_{0}, L_{0}\right\}$ meets both the inner common tangents of $K_{0}$ and $L_{0}$. In particular, no member of $\mathscr{K}$ is contained in the interior of that cone.

Denote the inner common tangents of $K_{0}$ and $L_{0}$ by $b_{0}$ and $c_{0}$ and their intersection point by $A_{0}$. The complement of the double cone above consists of two opposite open cones $R_{1}$ and $R_{2}$, say, bounded by $b_{0}$ and $c_{0}$ and separated by the common apex $A_{0}$. Let $\mathscr{K}_{1}$ and $\mathscr{K}_{2}$ be the subfamilies of $\mathscr{K}$ whose members lie entirely in $R_{1}$ and $R_{2}$, respectively. Of course, $\mathscr{K}_{1}$ or $\mathscr{K}_{2}$ may be empty. Each set in $\mathscr{K} \backslash\left(\mathscr{K}_{1} \cup \mathscr{K}_{2}\right)$ meets one of the lines $b_{0}$ and $c_{0}$ (or both). The following observation was already made by Kramer [12].

Let $g$ be a line through $A_{0}$. If $g$ separates two members of $\mathscr{K}_{1}$, say, then $g$ is a stabbing line for $\mathscr{K}_{2}$.

Suppose this is not true. Then, without loss of generality, $g$ separates two members $K_{1}$ and $L_{1}$ of $\mathscr{K}_{1}$ and misses some member $K_{2}$ of $\mathscr{K}_{2}$, where $K_{0}, K_{1}$, and $K_{2}$ lie in one of the closed half-planes bounded by $g$ and $L_{0}$ and $L_{1}$ lie in the other (see Fig. 2). It follows at once that $K_{1}, K_{2}$, and $L_{0}$ are separated by $b_{0}, c_{0}$, and $g$, even though it may happen that $L_{0}$ touches $g$ at the point $A_{0}$. This violates the hypothesis of a common line transversal for $K_{1}, K_{2}$, and $L_{0}$, and so the assertion is proved.

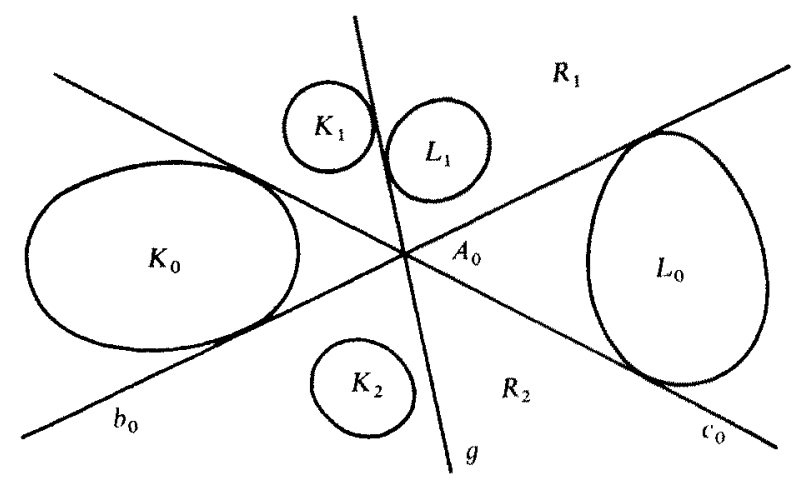

Fig. 2. Two members $K_{1}$ and $L_{1}$ of $\mathscr{H}_{1}$ separated by $g$. 
Assume, for the moment, that every two members of $\mathscr{K}_{1}$ admit a line transversal passing through $A_{0}$. Then $\mathscr{K}_{1}$ has a stabbing line through $A_{0}$. To see this, map the sets in $\mathscr{K}_{1}$ onto a suitable line which cuts through the cone $R_{1}$, using central projection with center $A_{0}$. By Helly's theorem on that line, the resulting segments have a common point and so the line connecting that point to $A_{0}$ is the desired stabbing line for $\mathscr{K}_{1}$. If, in addition, every two members of $\mathscr{K}_{2}$ admit a line transversal through $A_{0}$, then $\mathscr{K}_{2}$ also has a stabbing line passing through $A_{0}$. Consequently, each set in $\mathscr{K}$ meets $b_{0}$ or $c_{0}$ or one of the stabbing lines for $\mathscr{K}_{1}$ and $\mathscr{K}_{2}$. This would conclude the proof of our theorem.

For the remainder of the proof it therefore suffices to assume that one of the families $\mathscr{K}_{1}$ and $\mathscr{K}_{2}$ contains two members without a common transversal through $A_{0}$. In fact, it is enough to require that there is some line through $A_{0}$ which separates the two sets. Without loss of generality, suppose it separates two sets in $\mathscr{K}_{1}$.

Let $a_{0}$ denote the outer common tangent of $K_{0}$ and $L_{0}$ which cuts through the cone $R_{1}$, and let $B_{0}$ and $C_{0}$ be its intersection points with $c_{0}$ and $b_{0}$, respectively. To simplify the following discussion, we assume that the ordered triple $\left(A_{0}, B_{0}, C_{0}\right)$ is clockwise oriented and that $K_{0}$ touches $c_{0}$ between the points $A_{0}$ and $B_{0}$ (see Fig. 3). This can always be achieved by relabeling the pairs $b_{0}, c_{0}$ and $K_{0}, L_{0}$, if necessary. Denote the closed triangle with vertices $A_{0}, B_{0}$, and $C_{0}$ by $\Delta_{0}$. Every member of $\mathscr{K} \backslash \mathscr{K}_{2}$ not contained in the interior of $\Delta_{0}$ meets one of the lines $a_{0}$, $b_{0}$, and $c_{0}$.

We now proceed as follows. We rotate the line $c_{0}$ clockwise around $A_{0}$ until it is about to leave for the first time-some member of $\mathscr{K}_{1}$. Let $c_{0}^{\prime}$ denote the rotated line at this point, and let $K^{\prime}$ be such a member. Since $\mathscr{K}_{1}$ is assumed to be nonempty, $K^{\prime}$ and $c_{0}^{\prime}$ clearly exist. By construction, no member of $\mathscr{K}_{1}$ lies in the open wedge formed by $c_{0}$ and $c_{0}^{\prime}$ and contained in $R_{1}$. This means that $c_{0}^{\prime}$

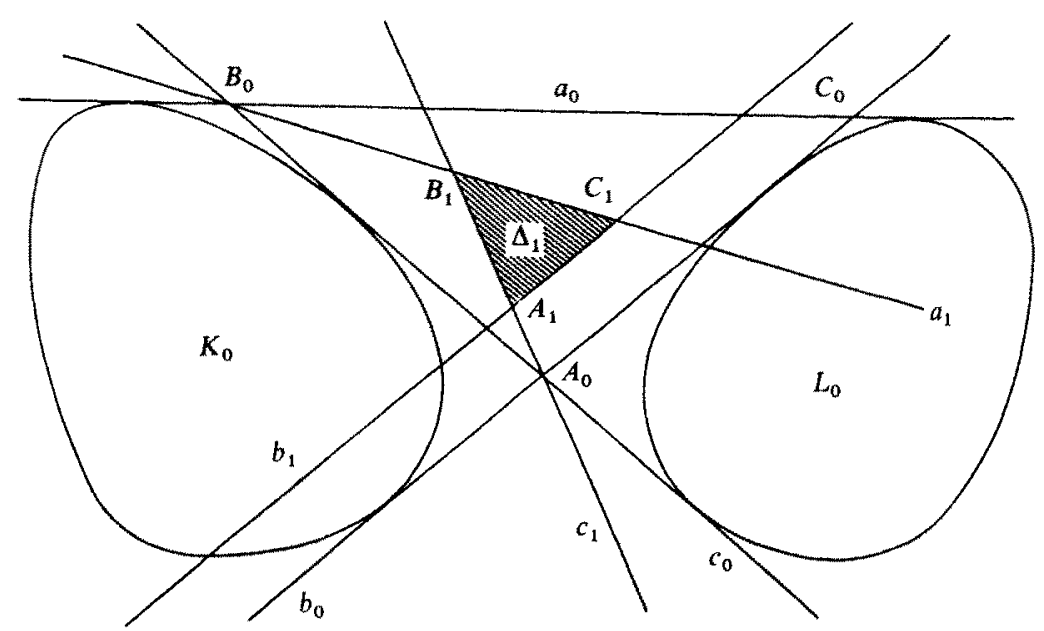

Fig. 3. The ordered triple $\left(A_{0}, B_{0}, C_{0}\right)$. 
must separate $K^{\prime}$ from some member of $\mathscr{K}_{1}$; otherwise, no two sets in $\mathscr{K}_{1}$ could be separated by a line through $A_{0}$, contrary to the assumption on $\mathscr{K}_{1}$ made earlier in the proof. Thus, by $(3,1), c_{0}^{\prime}$ is a stabbing line for $\mathscr{K}_{2}$. With $\Delta_{0}^{\prime}$ denoting the closed triangle formed by $a_{0}, b_{0}$, and $c_{0}^{\prime}$, the four lines $a_{0}, b_{0}, c_{0}$, and $c_{0}^{\prime}$ clearly intersect all the members of $\mathscr{K}$ except those lying in the interior of $\Delta_{0}^{\prime}$ (if such members exist at all).

We next apply a technique which was introduced by Kramer [12] and played a central role in this proof that $T(3)$ implies $T^{5}$. (A brief description of Kramer's approach is given in Section 4.) Since our goal here is to improve on Kramer's result by replacing $T^{5}$ with $T^{4}$, we find it necessary to modify and at the same time extend his method. The general idea is to move the lines $a_{0}, b_{0}$, and $c_{0}^{\prime}$ from their original position to a more tractable "extremal" position, making sure that the property stated in the last sentence of the preceding paragraph is preserved during the transition.

To be specific, we rotate $a_{0}$ about $B_{0}$ and $c_{0}^{\prime}$ about $A_{0}$, both in a clockwise direction, while translating $b_{0}$ toward $B_{0}$. We continue to do so as long as the equivalent of the above condition holds, that is as long as $c_{0}$ and the three moving lines intersect all the members of $\mathscr{K}$ except those lying in the interior of the triangle formed by the moving lines. For convenience, and only in the present context, the latter condition is called the "stabbing condition."

Two cases can occur.

In the first (and easy) case, the lines in question can be moved in such a way that the triangle formed by them degenerates, i.e., shrinks to a point, with the stabbing condition being satisfied all the time. Clearly, every member of $\mathscr{K}$ is then intersected by $c_{0}$ or one of the three versions of the moving lines passing through that point. Hence $\mathscr{K}$ can be stabbed by four lines, and we are done.

In the contrary case, no matter how the three lines are moved, they will always reach a position in which a nondegenerate triangle is formed, the stabbing condition is satisfied but fails if any of the lines is moved any further. This case is more difficult to analyze and occupies us for the rest of the proof.

Let $a_{1}, b_{1}$, and $c_{1}$ be versions of the original lines $a_{0}, b_{0}$, and $c_{0}^{\prime}$ which form a "limit triangle" as described above. (It may happen, of course, that $a_{1}=a_{0}$, $b_{1}=b_{0}$, or $c_{1}=c_{0}^{\prime}$.) Obviously, each of these lines must be "fixed" by some member of $\mathscr{K}$, or else we would be back to the first case. That is to say, there exist sets $K_{a}, K_{b}$, and $K_{c}$ in $\mathscr{K}$ such that $a_{1}, b_{1}$, and $c_{1}$ are tangent to $K_{a}, K_{b}$, and $K_{\mathrm{c}}$, respectively, while after a (small) further rotation of $a_{1}$ or $c_{1}$ or a further translation of $b_{1}$, the contact is lost; moreover, each of the sets $K_{a}, K_{b}$, and $K_{c}$ misses $c_{0}$ and intersects only the line having the same label.

It follows immediately that $K_{c}$ belongs to $\mathscr{K}_{1}$ or to $\mathscr{K}_{2}$ and that $K_{a}$ and $K_{0}$ lie on different sides of $c_{0}$. Indeed, if $K_{a}$ and $K_{0}$ were on the same side of $c_{0}$, then $K_{a}$ would have to intersect $b_{0}$ since otherwise the interior angle $\sigma\left(K_{a}, L_{0}\right)$ would be smaller than $\sigma\left(K_{0}, L_{0}\right)$, a contradiction. A fortiori, $K_{a}$ would have to intersect $b_{1}$; however, this is excluded by the choice of $K_{a}$.

Let $\Delta_{1}$ denote the closed triangle formed by $a_{1}, b_{1}$, and $c_{1}$, and let $A_{1}, B_{1}$, and $C_{1}$ be its vertices, where the labeling is such that $A_{1}$ lies across from $a_{1}$, and so on. We claim that $\Delta_{1}$ does not contain any member of $\mathscr{K}$ in its interior. This 
will conclude the proof, as is evident from the construction of $\Delta_{1}$. So assume, contrary to the claimed result, that there do exist members of $\mathscr{K}$ which lie in the interior of $\Delta_{1}$. Let $K_{1}$ be such a member. We then consider the six unbounded (closed) polygonal regions in the plane generated by the arrangement of lines $a_{1}$, $b_{1}$, and $c_{1}$ (see Fig. 3). For brevity, these regions are called pointed or blunt, depending on whether they intersect the triangle $\Delta_{1}$ in a single vertex or a full side. Each of the sets $K_{a}, K_{b}$, and $K_{c}$ lies entirely in one of these regions but of course a given region may contain more than one of the sets. We now show that of all the combinatorially distinct ways of assigning the sets to the regions, none can actually occur.

To this end, consider first $K_{a}$. It is clear that $K_{a}$ cannot lie in the pointed region with vertex $B_{1}$. For, otherwise, sets $K_{a}, K_{1}$, and $K_{0}$ would be separated by lines $a_{1}, c_{1}$, and $c_{0}$ and so would not admit a line transversal. (Here and in similar cases below we invite the reader to make the necessary verifications.) This is impossible. On the other hand, $K_{a}$ cannot lie in the pointed region with vertex $C_{1}$ either because this would mean that $\sigma\left(K_{1}, K_{a}\right)<\sigma\left(K_{0}, L_{0}\right)$, contrary to the assumption that the latter angle is minimal. Since $K_{a}$ and $K_{0}$ are separated by $c_{0}$, we conclude that $K_{a}$ is contained in the blunt region with vertices $B_{1}$ and $C_{1}$.

Next consider $K_{c}$. Recall that $K_{c}$ is a member of $\mathscr{K}_{1}$ or $\mathscr{K}_{2}$. If $K_{c} \in \mathscr{K}_{1}$, then, for the same reason as above, $K_{c}$ cannot lie in the pointed region with vertex $B_{1}$, Furthermore, it cannot lie in the pointed region with vertex $A_{1}$ since in that case $K_{c}, K_{1}$, and $L_{0}$ would be separated by $b_{0}, c_{1}$ and the line connecting $A_{1}$ and $C_{0}$, again a contradiction. If, on the other hand, $K_{c} \in \mathscr{K}_{2}$, then $K_{0}, K_{1}$, and $K_{c}$ are separated by the concurrent lines $b_{0}, c_{0}$, and $c_{1}$ (compare the proof of (3.1) above). Hence $K_{c}$ belongs to $\mathscr{K}_{1}$ and must be included in the blunt region with vertices $A_{1}$ and $B_{1}$.

Finally, what can be said about $K_{b}$ ? On the one hand, $K_{b}$ cannot lie in the pointed region with vertex $C_{1}$. This follows exactly as in the case of $K_{a}$ above. On the other hand, $K_{b}$ cannot lie in the pointed region with vertex $A_{1}$ either. Here the argument is a little different. Since $K_{b}$ is not intersected by $c_{0}$ we know that $K_{b}$ is either contained in the triangle formed by $b_{1}, c_{1}$, and $c_{0}$, or is separated from that triangle by $c_{0}$. In the first case, $K_{b}, K_{1}$, and $L_{0}$ would have no common line transversal, by the same reasoning as was used above for $K_{c}$. In the second case, $K_{b}, K_{c}$, and $L_{0}$ would fail to have a line transversal because they are separated by $b_{1}, c_{1}$, and $c_{0}$. Therefore, both cases cannot occur and we conclude that $K_{b}$ is contained in the blunt region with vertices $C_{1}$ and $A_{1}$.

To summarize the above discussion, it is found that the sets $K_{a}, K_{b}$, and $K_{c}$ lie one in each of the three blunt regions surrounding the triangle $\Delta_{1}$. By construction, none of the sets touches the two infinite rays on the boundary of its region. Hence $K_{a}, K_{b}$, and $K_{c}$ are separated by $a_{1}, b_{1}$, and $c_{1}$ and so do not admit a common line transversal. However, this is forbidden by the basic assumplion that $\mathscr{K}$ has property $T(3)$. It turns out, therefore, that the geometric situation described above cannot arise after all. That is, $K_{1}$ does not exist; in other words, the triangle $\Delta_{1}$ does not contain any member of $\mathscr{W}$ in its interior. This means that every member of $\mathscr{K}$ intersects $a_{1}, b_{1}, c_{1}$, or $c_{0}$, whence $\mathscr{K}$ can be stabbed by four lines. This completes the proof of our theorem. 


\section{Remarks}

1. We like to comment on the way in which the stabbing lines $a_{1}, b_{1}$, and $c_{1}$ are constructed in the foregoing proof. The reader has certainly noticed that our definition of these lines is not symmetric, in the sense that $a_{1}$ and $c_{1}$ result from a clockwise rotation of $a_{0}$ about $B_{0}$ and $c_{0}^{\prime}$ about $A_{0}$, respectively, while $b_{1}$ is obtained by translating $b_{0}$ toward $B_{0}$. Why cannot $b_{1}$ be a clockwise rotation of $b_{0}$ around $C_{0}$ ? The reason is that we want the set $K_{b}$ which fixes $b_{1}$ to be included in the blunt region with vertices $C_{1}$ and $A_{1}$. If $b_{1}$ were obtained from $b_{0}$ by rotating the latter instead of translating it, then it could happen that $K_{b}$ and $L_{0}$ lie on different sides of $b_{1}$. In that case, $K_{b}$ would be contained in the blunt region with vertices $B_{1}$ and $C_{1}$ and so $K_{a}, K_{b}$, and $K_{c}$ would not be separated by $a_{1}, b_{1}$, and $c_{1}$ any more. Hence the ensuing argument which leads to the nonexistence of $K_{1}$ would break down.

In principle, the same kind of problem could arise with the set $K_{a}$ which fixes $a_{1}$. However, here the situation is different, due to the fact that we chose $c_{0}$ to be one of the stabbing lines for $\mathscr{K}$. It was shown in the proof that the presence of $c_{0}$ forces $K_{a}$ and $K_{0}$ to be separated by $c_{0}$ and thus prevents $K_{a}$ from lying in the blunt region with vertices $A_{1}$ and $B_{1}$.

This seems a good place to point out the main differences between Kramer's method (as demonstrated in [12]) and the approach taken in this paper.

Kramer starts out with a pair $\left\{K_{0}, L_{0}\right\}$ having smallest interior angle $\sigma\left(K_{0}, L_{0}\right)$ and defines $a_{0}, b_{0}, c_{0}, A_{0}, B_{0}, C_{0}, \mathscr{K}_{1}$, and $\mathscr{K}_{2}$ just as we do here. He assumes, as he may, that there is some line through $A_{0}$ which separates two members of $\mathscr{K}_{1}$. He then goes on to show that $\mathscr{K}_{1}$ can be stabbed by three suitable lines $a_{1}$, $b_{1}$, and $c_{1}$. These lines are obtained in a symmetric fashion by rotating $a_{0}, b_{0}$, and $c_{0}$ clockwise about $B_{0}, C_{0}$, and $A_{0}$, respectively. Except for the fact that $b_{0}$ is rotated and not translated, the procedure is completely analogous to the one used in defining the corresponding lines in this paper. However, the necessary case analysis is much easier because all that has to be cared about is the sets in $\mathscr{K}_{1}$. It follows that every member of $\mathscr{K} \backslash \mathscr{K}_{2}$ intersects $b_{0}, c_{0}, a_{1}, b_{1}$, or $c_{1}$.

The last step in Kramer's proof consists in rotating the line $c_{1}$ "backwards," i.e., counterclockwise around $A_{0}$, until it is about to leave some member of $\mathscr{H}_{1}$ for the first time. Such a member must exist. Let $c_{1}^{\prime}$ denote the rotated line at this point, where of course $c_{1}^{\prime}=c_{1}$ is possible. As is readily seen, $c_{1}^{\prime}$ separates two members of $\mathscr{K}_{1}$ since otherwise no two members of $\mathscr{K}_{1}$ could be separated by a line through $A_{0}$; the latter was excluded above. Hence by $(3.1), c_{1}^{\prime}$ is a line transversal for $\mathscr{K}_{2}$. On the other hand, it is obvious that $a_{1}, b_{1}$, and $c_{1}^{\prime}$ still meet all the sets in $\mathscr{K}_{1}$. Consequently, every member of $\mathscr{K}$ is intersected by one of the lines $b_{0}, c_{0}, a_{1}, b_{1}$, and $c_{1}^{\prime}$. This completes Kramer's reasoning.

We gratefully acknowledge that without Kramer's pioneering work, this paper would not have been written.

2. As pointed out in the Introduction, the proof of our theorem (satisfying as it may be) leaves open the possibility that $T(3)$ might imply not only $T^{4}$, but even $T^{3}$. In fact, we believe that the latter is true although we cannot offer any supporting evidence for this except our inability to find a counterexample. 
Part of the difficulty seems to lie in the fact that $T(3)$ is a far weaker property than, say, $T(4)$. To justify this statement, let us briefly recall (from [3] or [4]) how we proved that $T(4)$ implies $T^{2}$. Consider a family $\mathscr{K}$ of compact convex sets in the plane. A result of Hadwiger and Debrunner (see p. 62 of [10]) asserts that if $\mathscr{X}$ satisfies $T(4)$, then there are two directions in the plane such that any two members of $\mathscr{K}$ admit a line transversal in one or the other direction. More generally, let us say that $\mathscr{K}$ has property $D_{m}(r)$, with $D$ representing direction, provided $m$ directions in the plane can be found such that any $r$ or fewer sets in $\mathscr{K}$ have a line transversal in one of these directions. (Transversal properties of this and a closely related type have been studied by Wegner [15].) Thus $T(4)$ implies $D_{2}(2)$. In turn, $D_{2}(2)$ can be shown to be strong enough to imply $T^{2}$.

It might be expected that similar (albeit weaker) conclusions can be established in the case when $\mathscr{K}$ has property $T(3)$. For example, it is plausible that $T(3)$ should imply $D_{3}(2)$. Since it is easily verified that properties $T(3)$ and $D_{3}(2)$ combined imply $T^{3}$, this would settle the problem. ${ }^{3}$ Unfortunately, $D_{3}(2)$ is not in general a consequence of $T(3)$. Wegner (personal communication) has constructed examples which show that $T(3)$ does not even imply $D_{m}(2)$, no matter how large an $m$ is chosen. For $m=3$, Wegner's example is illustrated in Fig. 4.

We must conclude, therefore, that the problem of finding the desired stabbing number cannot be tackled in the manner suggested by these (and similar) considerations. It seems safe to say that new tools and ideas are required to solve the problem.

3. Gallai-type problems for line transversals in the plane can, of course, be studied in any sufficiently large "ground family" of compact convex sets. Apart from the family of all such sets considered in this paper, the most interesting and natural examples are supplied by the family of all (positive) homothets and the family of all translates of a given compact convex set $K$. As already mentioned, the stabbing numbers for these families were first investigated by Hadwiger and Debrunner [10].

Again, in view of (1.1) and the fact that there is no Helly-type transversal theorem for translates, the only unresolved problem in this context arises for $r=3$. The following is known (see [4]):

$$
\begin{array}{ll}
T(3) \Rightarrow T^{4} & \text { for families of homothets of } K, \\
T(3) \Rightarrow T^{2} & \text { for families of translates of } K .
\end{array}
$$

Moreover, any family of translates of $K$ satisfying $T(3)$ can be stabbed by two parallel lines. Of course, the result for homothets is now superseded by the corresponding result for general convex sets, i.e., assertion (1.3). However, a direct proof is still of interest and, in fact, very easy. It proceeds as follows:

Let $\mathscr{K}$ be a family of homothets of $K$ having property $T(3)$. It may be assumed that $K$ has nonempty interior and that $\mathscr{K}$ is finite, hence contains a smallest

\footnotetext{
${ }^{3}$ Whether $D_{3}(2)$ alone is sufficient for $T^{3}$ is not known. It follows from a result of Gyárfas and Lehel [9] (see the note at the end of [15]) that $D_{3}(2)$ implies $T^{4}$.
} 


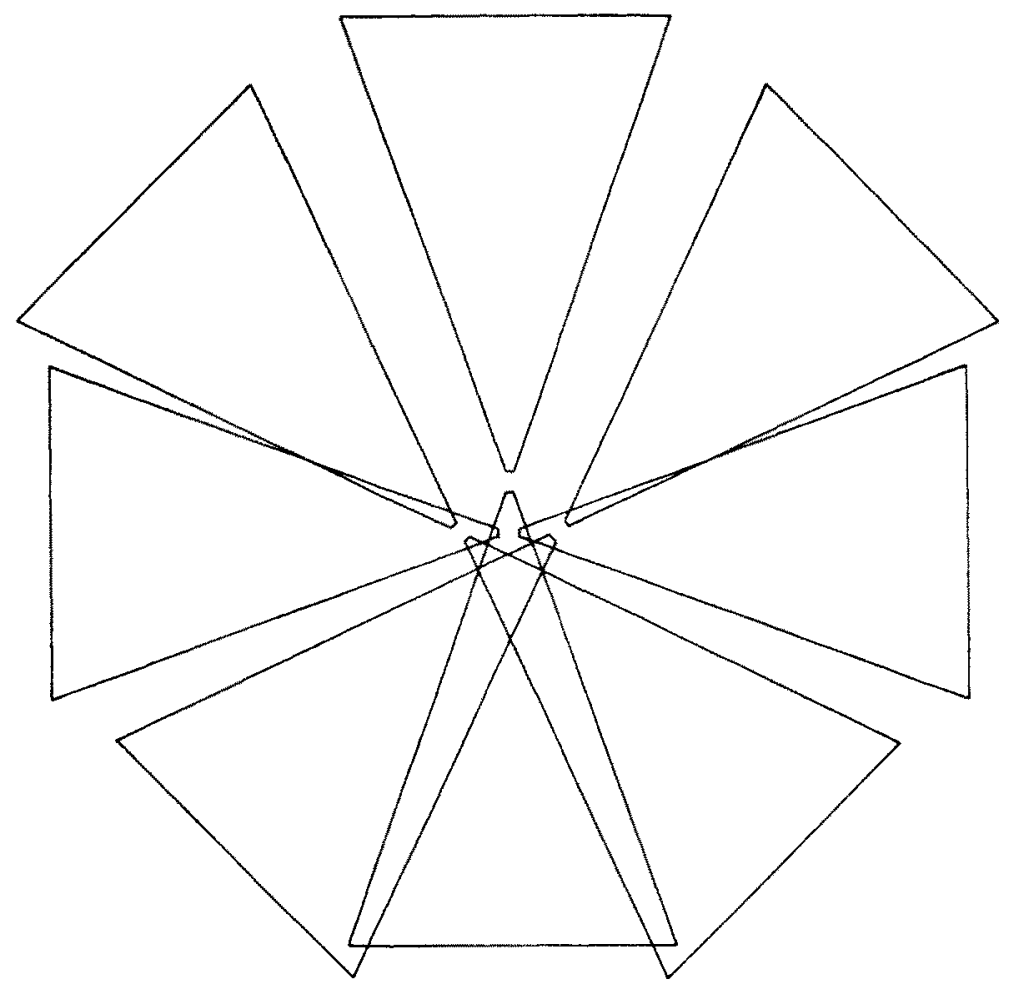

Fig. 4. Wegner's example.

homothet $K_{0}$, say. If every other member of $\mathscr{K}$ intersects $K_{0}$, then any two parallel supporting lines of $K_{0}$ suffice to stab $\mathscr{K}$. Otherwise, let $L_{0}$ be disjoint from $K_{0}$ and such that the interior angle $\sigma\left(K_{0}, L_{0}\right)$ defined in Section 2 is minimal. Then it is immediate that each set in $\mathscr{K}$ intersects one of the four supporting lines of $K_{0}$ which are laid out in the directions of the inner common tangents of $K_{0}$ and $L_{0}$. Thus $\mathscr{K}$ satisfies $T^{4}$, as claimed.

This straightforward argument is due to Wegner and was reported in [4]. It contrasts sharply with the rather complicated proof for general families of convex sets given in this paper. We believe that for any family of homothets of $K$, just as in the case of translates, $T(3)$ implies $T^{2}$.

\section{References}

1. L. Danzer, Uber ein Problem aus der kombinatorischen Geometrie, Arch. Math. 8(1957), 344-351.

2. L. Danzer, B. Grünbaum, and V. Klee, Helly's theorem and its relatives, in Convexity (Proc. Symp. Pure Math., Vol. 7), 101-180, American Mathematical Society, Providence, RI, 1963.

3. J. Eckhof, Transversalenprobleme vom Gallaischen Typ, Dissertation, Universität Göttingen, 1969.

4. J. Eckhof, Transversalenprobleme in der Ebene, Arch. Math. 24 (1973), 195-202. 
5. J. E. Goodman, R. Pollack, and R. Wenger, Geometric transversal theory, in New Trends in Discrete and Computational Geometry, J. Pach, ed., Springer-Verlag, Heidelberg, 1993.

6. P. M. Gruber and R. Schneider, Problems in geometric convexity, in Contributions to Geometry (Proc. Geom. Symp. Siegen 1978), 255-278, Birkhäuser-Verlag, Basel, 1979.

7. B. Grünbaum, On common transversals, Arch. Math. 9 (1958), 465-469.

8. B. Grünbaum, Common secants for families of polyhedra, Arch. Math. 15 (1964), 76-80.

9. A. Gyárfás and J. Lehel, A Helly-type problem in trees, in Combinatorial Theory and Its Applications, II (Colloq. Math. Soc. János Bolyai, Vol. 4), 571-584, North-Holland, Amsterdam, 1970.

10. H. Hadwiger, H. Debrunner, and V. Klee, Combinatorial Geometry in the Plane, Holt, Rinehart, and Winston, New York, 1964.

11. M. Katchalski, A conjecture of Grünbaum on common transversals, Math. Scand. 59 (1986), 192-198.

12. D. Kramer, Transversalenprobleme vom Hellyschen und Gallaischen Typ, Dissertation, Universität Dortmund, 1974.

13. L. A. Santaló, Un teorema sobre conjuntos de paralelepipedos de aristas paralelas, Publ. Inst. Mat. Univ. Nac. Litoral 2 (1940), 49-60.

14. H. Tverberg, Proof of Grünbaum's conjecture on common transversals for translates, Discrete Comput. Geom. 4 (1989), 191-203.

15. G. Wegner, Ein ebenes Transversalenproblem, Monatsh. Math. 77 (1973), 72-81.

Received September 13, 1990, and in revised form July 24, 1992. 